\title{
Philosophiques
}

\section{Duchesneau, F. L'empirisme de Locke, LaHaye, M. Nijhof, 1973, 261 pages.}

\section{Sylvain Auroux}

Volume 1, numéro 1, avril 1974

URI : https://id.erudit.org/iderudit/203007ar

DOI : https://doi.org/10.7202/203007ar

Aller au sommaire du numéro

Éditeur(s)

Société de philosophie du Québec

ISSN

0316-2923 (imprimé)

1492-1391 (numérique)

Découvrir la revue

Citer cet article

Auroux, S. (1974). Duchesneau, F. L'empirisme de Locke, LaHaye, M. Nijhof, 1973, 261 pages. Philosophiques, 1(1), 147-170. https://doi.org/10.7202/203007ar

Ce document est protégé par la loi sur le droit d'auteur. L'utilisation des services d’Érudit (y compris la reproduction) est assujettie à sa politique d'utilisation que vous pouvez consulter en ligne.

https://apropos.erudit.org/fr/usagers/politique-dutilisation/ 


\section{ÉTUDES CRITIQUES}

DUCHESNEAU, F. L'empirisme de Locke, LaHaye, M. Nijhof, 1973, 261 pages.

\section{par Sylvain AUROUX}

On manquait d'ouvrages en langue française consacrés à la théorie lockienne de la connaissance; cette lacune est en partie comblée par le livre de monsieur Duchesneau, à qui l'on devait déjà le chapitre Locke, de l'Histoire de la Pbilosopbie, dirigée par monsieur Chatelet ${ }^{1}$. Le but de l'auteur est, non pas d'étudier la théorie lockienne de la connaissance, mais plus précisément de déterminer la signification de l'empirisme de Locke; par là, il entend aussi apporter sa contribution à une définition de l'empirisme. S'agissant de l'auteur de l'Essay concerning Human Understanding, la première question ne peut être séparée de la seconde. Chacun, en effet, s'accorde pour voir en Locke un empiriste, et aussi pour opposer l'empirisme au rationalisme. Mais empiriste, Locke pourrait l'être simplement dans la perspective de «l'Experimental Philosophy» qui, de son temps, prévalait en Angleterre; en ce sens, serait empiriste celui qui considère l'observation comme la méthode la plus appropriée pour l'acquisition d'un savoir réel, et pas seulement verbal. Dans ce cas, peut-on opposer l'empirisme au rationalisme, l'un «comme une doctrine de la connaissance suivant laquelle les données de l'expérience, c'est-à-dire plus particulièrement de la perception sensible, sont aussi bien les éléments que les critères des jugements que nous formulons sur la réalité objective; » l'autre «comme la doctrine suivant laquelle la raison de l'homme est une fonction autonome de l'esprit qui pose, par un acte décisoire, les normes de l'ordre et de l'intelligibilité du réel $\gg$ ? On objecterait volontiers à monsieur Duchesneau que cette définiton du rationa-

«John Locke», Histoire de la Pbilosopbie. Idées, Doctrines, Tome IV : Les Lumières (le XVIIle siècle), Paris, Hachette, 1972, pp. 19-45. 
lisme a l'inconvénient de l'identifier à l'idéalisme, et plus particulièrement à l'idéalisme transcendental; il en résulte qu'elle est historiquement inopérante, ne pouvant pas même convenir au cartésianisme. Cette objection cependant a peu d'importance: l'auteur remplace la confrontation de l'empirisme et du rationalisme par celle des philosophies lockienne er cartésienne. La question générale qui vient d'être posée revient à démontrer dans quelle mesure il y a opposition radicale entre Locke et Descartes; or on peut voir, en recensant diverses interprétations des rapports entre les deux auteurs, combien le problème est délicat. Le 18 mars 1690, Tyrrel écrit à Locke qu'à Oxford, l'auteur de l'Essay passe pour être un disciple de Descartes. L'évêque de Worcester (Discourse in Vindication of the Doctrine of the Trinity, pp. 227-228) l'accuse d'emprunter à Descartes la notion d'évidence fondée sur les idées claires et distinctes ${ }^{2}$. Leibniz voit en lui un gassendiste, tandis que les Anciens considèrent essentiellement son opposition à Descartes. Les historiens du XIXe siècle (Webb, Harteinstein, Geil, Von Hertling, Gibson) créeront l'image d'un Locke trouvant l'origine de sa réflexion dans la métaphysique cartésienne, et en développant la critique. L'originalité du travail de monsieur Duchesneau est d'envisager la question du point de vue de l'histoire interne des doctrines; de montrer que l'origine de la réflexion lockienne se situe à l'extérieur du cartésianisme, et que «par conséquent, s'il se

2. Le recours à Stillingfleet nous paraît quelque peu spécieux: l'évêque de Worcester a trop intérêt à tirer, sur ce point, Locke vers Descartes, pour que son opinion puisse être prise en considération; ce qui l'oppose à Locke, c'est le chap. iii, 6, du livre IV de l'Essay, où est admis que notre connaissance soit plus bornée que nos idées; ainsi, bien que nous ayons des idées de matière et de pensée, nous ne serons peut-être «jamais capables de comprendre si un être purement matériel pense ou non ».

A quoi le savant prélat s'indigne et répond par l'application du principe cartésien «tout ce gui est renfermé dans l'idée claire et distincte d'une chose peut en être affirmé avec vérité ». Si Locke avait le même critère de l'évidence que Descartes, il serait mis en contradiction, d'où le rôle tactique de l'affirmation de l'évêque. Les disciples français de Locke ont bien vu l'importance de ce rejet du principe cartésien, cf. Condillac, Traité des Systèmes, VI, 2 : «Tous ceux qui sont un peu versés dans la lecture des ouvrages philosophiques remarqueront aisément combien de chimères naissent de ce principe: tout ce qui est renfermé dans l'idée claire et distincte d'une chose en peut être affirmé avec vérité ». 
rencontre avec Descartes ou s'oppose à lui, c'est moins par suite de divergences à partir d'une même orientation, que par l'incidence de problèmes analogues $»$. De la méthode choisie, découle l'ordre général de l'ouvrage, ainsi que l'orientation des recherches qu'il expose $;^{3}$ les sources et les déterminations de l'empirisme lockien doivent être cherchées dans les premiers écrits et les premières influences du philosophe, le rapport au cartésianisme dans les prises de positions de Locke à l'égard de celui-ci.

1 - Locke étudie la médecine avant de connaître le cartésianisme puisque, si on trouve des références médicales dans ses carnets de notes dès 1652 , on n'en trouve faites à Descartes qu'en 1660. Boyle (Sceptical Chymist, 1661) oriente les premières recherches scientifiques, c'est Sydenham (Metbodus curandi febres, 1666; Observationes Medicae, 1676) qui exerce la plus grande influence; Locke fut son collaborateur et son disciple. En remarquant le rôle de Sydenham, monsieur Duchesneau suit la tradition des historiens modernes de la philosophie $;{ }^{4}$ son apport personnel consiste à montrer comment les rapports de Locke avec la médecine ont évolué, de la rencontre avec Sydenham jusqu'aux positions philosophiques de l'Essay. Le premier chapitre, intitulé « Locke et la Doctrine de Sydenham » est essentiellement consacré à l'exposition de la méthodologie de ce dernier ${ }^{5}$. La méthode sydenhamienne repose essentiellement sur l'observation exacte des maladies et l'établissement d'une méthode de traitement précise et régulière; elle emprunte en cela à l'histoire baconienne, et aboutit à la critique des hypothèses, c'est-à-dire au rejet des explications a priori de la réalité. Il y a chez lui un certain phénoménisme; la théorie des constitutions morbides, notamment, envisage la maladie sous son apparence globale (climat, atmosphère, etc...) plutôt que d'en chercher les causes

3. Cf. Préface, p. X : «Notre recherche sur la signification de l'empirisme de Locke implique (...) qu'avant toute interrogation, nous examinions les faits qui permettent un rapprochement historique entre philosophies de Locke et Descartes 》.

4. Cf. BREHIER (P.U.F.) t. II, fasc. I, p. 241.

5. Signalons que monsieur Duchesneau est l'auteur d'un article sur \& la philosophie médicale de Sydenham», paru in: Dialogue, IX (1970), pp. 54-68. 
physiques cachées, par exemple, dans la théorie corpusculaire des corps. Le langage de Sydenham, losqu'il parle de causes réelles, d'effort, de la Nature, de matière morbifique, n'est pas cependant celui d'un empiriste qui réduirait toute connaissance aux données sensibles; il admet pourtant le relativisme de nos connaissances. Mais la technique médicale nécessite pour son succès la supposition d'un ordre régulier et invariable des phénomènes naturels; d'où le recours à un concept multiforme de nature, d'origine hippocratique (sur le développement des maladies), voire à un providentialisme théologique (chaque maladie possède son remède spécifique). La doctrine de Sydenham consiste à « limiter l'activité de médecin à investiguer minutieusement l'histoire des maladies et les effets des remèdes, suivant ce qu'enseigne le seul vrai maître, l'expérience ». Dans ses considérations sur l'essence des maladies, le médecin soutient que les symptômes finalement ne sont jamais que les signes généraux des effets indéfiniment variables de la nature. La méthode historique s'allie à la thèse de la rationalité du réel, pour permettre que la collation de symptômes spécifiques donne à connaître l'essence normale des affections. C'est cette méthodologie aux résonances tout à fait particulières, comme le montre par exemple la comparaison avec Robert Boyle, qui serait à la source de la réflexion du philosophe de l'Essay; monsieur Duchesneau analyse dans son second chapitre l'empirisme médical de Locke, c'est-à-dire les rapports de Locke et de Sydenham, et les écrits médicaux du premier. Après discussion de la thèse soutenue par monsieur Romanell $^{6}$ (réciprocité de la collaboration): l'auteur conclut qu' «en fonction des données historiques que nous possédons, nous sommes portés à concevoir le rôle de Locke comme celui d'un collaborateur perspicace, qui aurait parfois aidé le maitre à mettre ses idées au clair, qui aurait suggéré des modifications dans la présentation savante de la doctrine sydenhamienne $»$. Ceci permet à l'auteur de ne pas s'arrêter sur le problème de l'attribution à Locke du De Arte Medica (1669) et les fragments sur l'Anatomie (1668), et de passer directement à leur analyse ${ }^{7}$.

\footnotetext{
6. «Locke and Sydenham : a fragment on Smallpos Bulletin of the History of Medecine, Tome XXXII (1958) pp. 233-321.

7. L'attribution à Locke est traditionnelle (cf. Fox Bcurne, The Life of $J$.
} 
Pour Locke, d'après le plus ancien de ces textes, le but du savoir médical est de prolonger la vie, de la libérer des infirmités et des souffrances «as much as the constitution of our frail composure is capable of $»$. La médecine est un art et ce sont les règles et les méthodes de traitement qu'il s'agit d'amener à la certitude. Mais l'action ne peut s'appuyer que sur de multiples observations, au demeurant trop nombreuses pour reposer sur l'expérience d'un seul individu. Une critique des anciens, de la méthode hypothétique - le raisonnement hypothétique, est conçu ici comme raisonnement a priori - repose sur le principe qu'on ne peut aller au-delà de l'observation, que les causes dernières nous sont à jamais cachées, et que seules certaines relations causales peuvent être constatées. Locke nomme ces dernières causes extérieures et visibles par opposition aux causes internes et imperceptibles. La certitude du traitement tient à son succès, mais elle s'accommode d'un certain relativisme: "Quiconque aura amené la cure d'une maladie à une certitude approchant celle qui tient à l'alimentation d'un homme en santé à l'aide de quelque sorte de repas consistant que ce soit, peut se sentir autorisé à entreprendre avec confiance le traitement de cette espèce de désordre, et dans cette partie, il pourra avoir perfectionné l'art de la médecine, bien que peut-être, dans quelques cas réfractaires et irréguliers, sa méthode bien constituée puisse faillir et la maladie frustrer tous ses efforts de la réussite». Tout traitement cependant nécessite la régularité et la rationalité des symptômes pathologiques. C'est en ce point, sans doute, que Locke est original ; Hippocrate joint la méthode d'observation à une théorie finaliste de la nature (cf. la théorie de la prognose),

Locke, t. I, pp. 222-227; mais K. Dewburst (Dr. Thomas Sydenbam 1624-1689). His life and original writings, 1966) range les textes dans les oeuvres de Sydenham. Dans la note 104 de la page 41, monsieur Duchesneau signale en outre les travaux en cours du Pr. Bates sur ce problème, et justifie ainsi sa position: «nous croyons, pour notre part, qu'il a existé, dès le départ, une réciprocité d'apport intellectuel dans l'association Sydenham-Locke. Ainsi ne pensons-nous pas faillir à la vérité historique en traitant le De Arte Medica et l'Anatomie comme des traités sydenhamiens de Locke, en y cherchant l'un des motifs initiaux de l'empirisme de Locke». L'expression «réciprocité d'apport intellectuel» semble coïncider avec la thèse de Romanell, mais on peut la comprendre dans les limites définies précédemment. 
la signification des phénomènes pathologiques étant inscrite dans le cours des événements naturels. Sydenham interprète les symptômes selon l'expérimentalisme baconien, mais recourt à l'hippocratisme pour donner un sens aux phénomènes (finalité naturelle dans le développement de la maladie et le choix des remèdes, quoique sa science médicale soit un démenti partiel de sa position métaphysique). La thèse de monsieur Duchesneau (cf. p. 67) est que Locke reprend cette problématique téléologique en la plaçant, non dans l'ordre immanent à la nature, mais dans l'esprit de l'observateur. Ce qui l'intéresse, c'est la «technique humaine» de la médecine, «ce pourquoi il entreprend un examen psychologique et épistémologique des théories médicales. Sydenham accepte la doctrine hippocratique de la nature, mais sa méthodologie baconienne introduit à ce naturalisme; Locke saist l'originalité de la science sydenhamienne, il la comprend sans se référer à la philosophie de la nature d'Hippocrate, mais "pour comprendre la nouvelle science médicale dans sa constitution épistémologique, il introduit (...) l'équivalent de la prognose d'Hippocrate, dans une analyse de l'esprit ». L'auteur en conclut (ibid.) que la «thèse philosophique qui pourrait être l'expression de ce point de vue consisterait à postuler une doctrine de l'harmonie naturelle et de la rationalité propre à la connaissance, à l'arrière plan d'une analyse historique des idées et des choses :> . Pour vérifier ce point de vue, dans lequel réside l'originalité principale de son ouvrage,monsieur Duchesneau entend chercher s'il y a dans la doctrine médicale de Locke un développement de cette thèse, et si on en trouve la justification dans la philosophie de l'Essay. L'analyse du fragment sur l'Anatomie qui achève ce chapitre, montre en fait que Locke avait initialement des vues sceptiques sur la connaissance. Si le modèle mécaniste eșt le « workmanship of nature », et correspond à des processus élémentaires de type corpusculaire imperceptibles, en l'absence de distinction entre qualités premières et qualités secondes, toute connaissance est phénoménale. La connaissance des qualités sensibles (même lorsqu'elle fait appel au microscope, c'est en cela que cette épistémologie d'origine sydenhamienne se différencie de l'expérimentalisme philosophique de Bacon et Boyle) ne peut conduire à la connaissance de la réalité, et la recherche causale n'a pas de terme. Pourtant, sur le plan des phénomènes perceptibles, l'action 
des causes est compréhensible: le rapport des causes premières à notre connaissance est donc nécessairement lié à une finalité, d'autant que si les causes essentielles sont réputées inconnaissables, elles sont considérées comme existant réellement au plan des réalités extérieures. A cette époque, Locke n'a pas encore réfléchi sur le problème épistémologique ainsi posé ; pour ce faire, il faut passer de l'empirisme médical à l'empirisme philosophique.

2 - Même si l'on admet que l'empirisme de l'Essay dérive en grande partie des positions de Locke médecin, sur le plan philosophique, Locke demeure dans la tradition des théories empiristes du XVIle siècle, essentiellement celles de Gassendi et Hobbes. Ce sont ces deux doctrines que l'auteur analyse dans le troisième chapitre, après avoir montré les rapports historiques de Locke avec le gassendisme (par l'intermédiaire de Boyle, Willis, Bernier, Launay) ${ }^{8}$. Ces analyses demeurent cependant assez extérieures aux développements précédents; elles concernent plus les doctrines de Hobbes et Gassendi que ce que Locke en a pu tirer. Le but premier de l'auteur est de montrer que l'empirisme aboutit à un grave problème qu'il ne peut résoudre; pour Hobbes comme Gassendi, les conditions du savoir sont explicitées dans une théorie sensualiste de l'idée, mais la science trouve son fondement dans le modèle euclidien de la rationalité (cf. la réduction hobienne de la logique à un calcul) : «dès lors com-

8. Pourquoi cette question est-elle laissée de côté en ce qui concerne Hobbes? S'il est évident que Locke dans ses écrits politiques combat Hobbes, et possède une certaine connaissance de ses théories, il ne serait pas inutile de savoir ce qu'il en avait lu, dans quelles circonstances, et sur quels points précis, il pourrait y avoir influence de l'auteur du Léviatban. M. Duchesneau note que «Hobbes ne pourrait que confirmer Locke dans son phénoménisme» (p. 129), qu' «il est indubitable qu'il a suggéré à l'auteur de l'Essay le passage sceptique sur les rapports de l'âme et du corps» (p. 130), que «la doctrine du langage de l'Essay est très voisine de celle de Hobbes» (p. 131), mais tous ces rapprochements demeurent vagues. On peut notamment critiquer le rapprochement des deux théories du langage; pour le nominalisme hobésien, il n'y a pas d'idée générale mais des désignations de désignation (intention seconde) et l'arbirraire du langage rejaillit sur la vérité, comme Leibniz n'a cessé de le lui reprocher; la doctrine lockienne de l'abstraction est d'une inspiration totalement différente, et la vérité concerne l'adéquation de la pensée, y compris l'idée générale, aux choses. 
ment concevoir (...) la rationalité propre du réel et notre pouvoir de déduction? » En mettant au jour ce problème, l'intention de l'auteur est de montrer que Locke, lui, a trouvé une solution. On peut cependant formuler diverses réserves. Tout d'abord, n'est-ce-pas voir les doctrines empiristes au travers du rationalisme métaphysique, ou même de l'image que nous en donne le kantisme? Le problème de l'intelligibilité du réel a-t-il obligatoirement un sens pour l'empiriste? Si je pose que toutes mes idćes viennent de l'action du monde sur moi, qu'y a-t-il d'étonnant à ce quelles lui correspondent? La seule difficulté, c'est le mécanisme de cette correspondance, et à défaut d'hypothèses physiologiques, on peut se contenter de son affirmation comme simple fait ; le problème de la constance des phénomènes-lois n'en est peut-être pas un non plus, au niveau épistémologique du noins, car dans la perspective empiriste, c'est parce que les phénomènes sont constants que nous pensons qu'ils le sont, et non l'inverse; c'est parce que le réel cause en nous la pensée qu'il est pensable. On peut évoquer contre l'empirisme le problème de l'induction; je ne peux pas déduire $(x), f(a)$, $f(b)$, etc ..., en admettant que ces derniers sont des données sensibles; mais, inversement, on peut montrer que quiconque n'a pas eu comme données $f(a), f(b)$, etc... ne possède pas $(x)$ $\mathrm{f}(\mathrm{x})^{9}$. Il s'agit d'un problème différent de celui posé par l'intelligibilité du réel, et qui peut trouver sa solution dans le nominalisme; on remarquera que l'empirisme classique tient compte de la seconde partie du problème, et résoud la première dans les termes de sa théorie de l'abstraction ${ }^{10}$. Dans ces con ${ }^{-}$ ditions, présenter les philosophies de Gassendi et surtout de Hobbes comme le fait monsieur Duchesneau, nous paraît une thèse contestable sur la valeur intrinsèque. Dans la mesure où Hobbes ne pose pas le problème de la rationalité du réel, on ne peut lui imputer de ne l'avoir pas résolu; il nous faut, au contraire, comprendre comment son mécanisme, appliqué à la

9. Cf. B. RUSSELL, Human Knowledge, its scope and limits, particulièrement le dernier chapitre, "the limits of empiricism».

10. Si l'idée abstraite est l'idée concrète séparcée de ses déterminations, l'universalité est donnée dans la sensation, le problème ne se pose pas pour Hobbes, puisque la généralité et non l'universalité n'est qu'une dénomination arbitraire. 
connaissance, l'exclut. Par contre, puisque Locke utilise incontestablement un concept de finalité pour exposer l'accord de la pensée et du monde, ${ }^{11}$ il faut que l'historien de la philosophie explique la présence de ce thème en son oeuvre; ce que fait parfaitement monsieur Duchesneau, et de façon qui n'admet pas de réplique en montrant le rôle de l'empirisme médical dans la genèse des théories de l'Essay.

3 - Dans l'ensemble, le reproche qu'on pourrait faire à monsieur Duchesneau, outre son appréhension «rationaliste 》 de l'empirisme, c'est de considérer que Hobbes et Locke participent à une même problématique philosophique. Ce point de vue lui sert de fil conducteur pour l'analyse des «positions initiales de Locke sur le problème de la connaissance » qui constitue le chapitre IV de son ouvrage ${ }^{12}$. On le suivra donc dans cette voie. Si l'auteur reprend la thèse de M. Romanell (P.C.) selon laquelle le philosophe généralise l'emploi d'une méthode que Sydenham n'appliquait qu'à l'étude des maladies, il ajoute que l'empirisme médical, et l'empirisme gnoséologique n'offrent pas le même type de réduction: l'un réduit la science du réel à celle des phénomènes, l'autre le savoir à une origine sensible. Le passage s'est effectué rapidement chez Locke: les textes médicaux datent de 1668-1669, les deux premières esquisses de l'Essay de $1671^{13}$. Dès ces premières esquisses, il est affirmé que les qualités sensibles sont les plus simples idées que nous

11. Cf. Essay II, xxiii, 12 «Dieu qui, par sa sagesse infinie, nous a faits tels que nous sommes, avec toutes les choses qui sont autour de nous, a disposé nos sens, nos facultés et nos organes de telle sorte qu'ils puissent nous servir aux nécessités de cette vie, et à ce que nous pouvons avoir à faire dans ce monde $\gg$.

12. «Gascendi et Hobbes avaient soulevé un problème épistémologique que leur système ne leur permettait pâs de résoudre. Le problème concernait surtout la relation à établir entre les données empiriques de la connaissance, les idées fournies par les sciences, et les fondements d'une science certaine de la nature $(\ldots)$ Quelle est la théorie de la connaissance que Locke développe dans les esquisses de 1671 , en relation avec la problématique issue des systèmes de Hobbes et Gassendi, et avec les positions méthodologiques de la médecine sydenhamienne? »

13. On dispose de trois esquisses de l'Essay, désignées habituellement par A (Aaron et Gibb, Oxford, 1936), B (Rand, Harvard, 1931), C (inédite à la Pierpont Library de New York, et qui date de 1685). 
ayons, et les premiers objets de notre entendement. L'adéquation de l'idée et de la qualité sensible est comprise comme une ressemblance objective (image du miroir) mais Locke se refuse à examiner les bases matérielles ou spirituelles de la perception. L'idée de substance pose alors un grave problème. Toute qualité sensible d'une substance donnée semble, a priori, être déterminée par une dualité de relation: relations à l'essence de la chose dont elle est la propriété, relations aux autres qualités de la même substance. Quelle que soit la constance des relations phénoménales, puisque les idées simples ne contiennent pas en ellesmêmes la raison d'être des corrélations de l'expérience, la liaison subjective de diverses qualités sensibles ne saurait renvoyer à une liaison objective susceptible d'être perçue. Ia supposition que l'idée d'une substance est composée de l'association de diverses idées de qualités sensibles, présente certaines difficultés. L'expérience nous montre qu'il y a une modification des qualités sensibles suivant les causes en présence. Ce qui conduit Locke à infléchir sa théorie de la substance dans le sens de la causalité. Dès lors, l'idée n'est pas simplement une image, elle renvoie à une cause productive, et par qualité, il faut entendre toute chose existant hors de nous, et qui produit en nous une idée simple. Quant à la chose, il faut distinguer ses qualités actuelles et ses qualités potentielles; les qualités actuelles sont permanentes, et les qualités potentielles sont toutes les altérations qu'une chose peut produire dans une autre chose. Dès lors, ne doit-on pas concevoir les qualités actuelles comme productrices des autres, et leurs idées comme possédant une réalité objective? C'est à ce niveau que monsieur Duchesneau réintroduit la problématique qui oriente son travail : on retrouve le problème posé par l'existence de la science. Les matériaux de la connaissance sont fournis par les idées simples et complexes (dont certaines, comme celles des substances, nous sont imposées); il semble que l'expérience soit la source productrice à la fois des idées et de leur corrélation rationnelle. L'auteur en conclut que, si l'expérience est le fondement de la rationalité, c'est que le phénomène possède une rationalité interne, comme dans l'empirisme médical lockien. Si l'expérience peut donner la certitude en ce qui concerne l'existance, reste le problème des mathématiques; c'est d'une expérience proprement sensible de proportion constante entre des 
idées simples d'étendue ou de nombre que la connaissance mathématique tire sa certitude; l'esprit accorde d'abord sa certitude à ces proportions constantes, et en les appliquant aux données de l'expérience, il en tirerait leur rationalité. Monsieur Duchesneau note que, si l'étude historique des idées, de leurs combinaisons et relations, est d'une importance centrale, et donne lieu à une «logique nouvelle» ${ }^{14}$ pour l'utilisation des idées de la connaissance, «le problème de la rationalité du réel ne semble pas pouvoir être analysé strictement à ce niveau descriptif » (p. 160). Ce qui semble lui manquer «c'est essentiellement une doctrine de l'inférence ${ }^{15}$ capable d'établir les relations qui existent entre les déductions rationnelles et la rationalité propre du réel, telle qu'elle se manifeste à l'expérience ». Si on doit reconnaître que le but de Locke est essentiellement pratique "pouvons-nous concevoir de réduire toute (sa) philosophie de la connaissance (...) à la stricte méthode empiriste de description des données observables dans la connaissance? » Monsieur Duchesneau répond que c'est impossible et qu'il y a chez Locke une ligne métaphysique générale; il tente d'expliquer cette ligne en analysant le texte, reste manuscrit, des Essays on the Law of Nature (1664). $\mathrm{La}$ loi de la nature est l'expression de la volonté divine, mais le volontarisme lockien est limité : la loi doit s'interpréter en fonction de la structure ontologique du monde et de la liberté de l'homme, elle-même inscrite dans la structure téléologique de la nature. L'obligation de la loi de nature dépend matériellement du pouvoir qu'a l'homme de connaître la volonté divine par raison naturelle, ou par la révélation. Se pose le problème de savoir comment cette loi de nature nous est connaissable, d'au. tant que Locke développe une critique des idées innées, ou plutôt de l'inscription native de la loi dans l'esprit de l'homme; les arguments des Essays sont déjà ceux de l'esquisse B de 1671, et du livre premier de l'Essay de 1690, à ceci près qu'en 1664, est

14. Il nous semble que ceci mériterait plus ample développement; en quoi la logique lockienne ast-elle nouvelle? Nous aurions plutôt tendance à dire que la combinatoire lockienne des idées diffère peu de celle de Descartes, Port-Royal, et plus tard Crousur; ce qui est nouveau c'est la méthodologie des sciences; voir ici note 2 , et in fine notre conclusion.

15. Le mot nous paraît mal choisi, aucune théorie de l'inférence n'a jamais traité de ce problème, qui est une question d'ontologie. 
utilisé le terme «inscripta » pour définir la loi qui serait proprement gravée en nous de façon explicite. Il ne refuse cependant pas, comme le fait remarquer $M$. Polin ${ }^{16}$, le point de vue suivant lequel la loi de nature serait innée à notre nature de façon essentielle, bien qu'on ne puisse la lire immédiatement, ni même directement dans l'esprit de l'homme. Pour expliquer la doctrine de la connaissance que cela implique, monsieur Duchesneau fait appel à un texte du Journal, datant du 26 juin 1681, et à une comparaison avec le célèbre texte de Leibniz «Meditationes de Cognitione, Veritate et Ideis» (1684). Dans son Journal, Locke distingue deux sortes de connaissances, l'une générale, fondée sur les «idées justes et vraies» (right and true Ideas), l'autre particulière, fondée sur les faits ou la description des faits. C'est pour expliquer la notion lockienne d'idée vraie que monsieur Duchesneau fait appel à Leibniz. Arrêtons-nous à la notion leibnizienne d'adéquation: une notion est adéquate lorsqu'elle est une notion distincte, dont tout le contenu est connu. distinctement par analyse; «déterminer » nos idées consiste à pousser la définition jusqu'aux éléments irreductibles, ce qui n'est possible qu'à un certain degré pour la connaissance humaine, pour laquelle les déterminations se manifestent dans l'expérience; c'est pourquoi la connaissance mathématique ne concerne que la possibilité; il n'y a cependant nul doute que l'expérience signifie l'intelligibilité des causes réelles des phénomènes. Monsieur Duchesneau note que Locke serait d'accord pour reconnaître que les idées primitives sont celles dont l'intelligibilité ne peut être rendu distincte par analyse. Leibniz pose que ces idées primitives ne sont autre chose que les attributs de Dieu, Locke pose l'existence de Dieu comme la prémisse absolue qui fonde la possibilité des déductions morales. Notre connaissance ne peut qu'exprimer inadéquatement l'ordre du réel, à moins que le symbole, l'idée même ne devienne archétype intelligible, comme c'est le cas pour les vérités mathématiques. Locke ne se distingue de Leibniz que sur le problème de l'inscription originelle des vérités dans l'esprit humain. Monsieur Duchesneau en conclut à une dualité d'orientation de la première philosophie

16. «La politique morale de J.L.», p. 102, cf. "Essays on the Law of Nature $\gg$ I, p. 116. 
lockienne de la connaissance, l'analyse historique des éléments de la connaissance se double d'une entreprise de justifier déductivement les déterminations morales de l'action humaine. Avant de poursuivre l'analyse de l'ouvrage, nous aimerions faire quelques remarques. Tout d'abord, on peut se demander si la notion de loi de nature, autrement dit la philosophie du droit, est bonne pierre de touche pour juger la philosophie lockienne de la connaissance. Si l'empirisme a une origine médicale, la philosophie morale ainsi conçue a son origine chez les juristes. L'opposition entre la conception de la loi naturelle, telle qu'elle se développe depuis Grotius, Pufendorff, jusqu’à Barbeyrac et Burlamaciui, et une épistémologie empiriste est flagrante; Cassirer l'a noté pour montrer que le rationalisme éthique prédomine du XVIIe au XVIIIe siècle ${ }^{17}$. Pour résoudre cette opposition, certains partisans du droit naturel ont développé une théorie dite l'acquisition originaire qui rappelle celle de Locke ${ }^{18}$. On attendrait donc que soit mis en lumière le rôle des sources lockiennes, en ce qui concerne la doctrine morale, comme monsieur Duchesneau l'a fait pour une doctrine épistémologique en la rapportant à Sydenham. Il y a peut-être dans l'oeuvre de Locke la convergence de deux univers de discours hétérogènes. On pourrait contester aussi la valeur historique de la démarche effectuée dans ce chapitre: on utilise un texte de 1664 pour expliquer celui de 1671, et un texte de 1681 pour expliquer celui de 1664 ; il aurait fallu au moins prouver qu'on pouvait le faire. Quant à la notion d'idée juste et vraie, (right and true idea), on aurait aimé la voir comparer avec la doctrine de l'Essay; Locke y soutient que ${ }^{19}$ seules les propositions sont vraies ou fausses (true or false), et réserve aux idées les termes de «right or wrong», que Thurot traduisait excellemment par «justes ou fautives » $20 \mathrm{La}$ comparaison avec Leibniz appelle le même genre de remarque: le chapitre XXXI du Livre II de l'Essay est consacré à l'analyse des notions d'idées complètes et incomplètes, qui ressemblent

17. Cf. Die Pbilosopbie der Aufklärung, t. f. (Fayard, 1966), p. 247.

18. VAN BICMA, in L'espace et le Temps chez Leibniz et Kant, Alcan, 1908, cite p. 204, à ce propos, un texte intéressant.

19. Cf. II, xxxii, chap. intitulé en référence à Arnauld « Des Vraies et des Fausses Idées $\gg$.

20. Oeuvres de Locke et Leibniz (Didot, 1839) p. 234. 
d'assez près aux concepts leibniziens, idées adéquates et inadéquates. Mais les auteurs divergent sur le problème de savoir quelles idées sont complètes; pour Locke, les idées simples le sont. Leibniz refuse (Nouveau Essais, ad loc.) de considérer que les idées des qualités sensibles puissent l'être ${ }^{21}$. Cette divergence oppose radicalement l'empirisme et le rationalisme, et pose le problème de savoir ce qu'est l'idée simple pour Locke. A notre sens, il ne suffit pas de dire qu'elle est idée d'une qualité sensible, la question se pose de dire si elle est un universel ou un particulier. On peut admettre que toute sensation est particulière; ce que je sens est telle ou telle qualité sensible; mais pour Locke, l'idée générale (qui peut être simple au sens de non composée) est abstraite à partir des données de la perception qui conséquemment sont composées. Auquel cas, si l'idée de qualité sensible n'est pas composée, alors elle n'est pas une donnée immédiate de la sensation, et si elle est une donnée immédiate de la sensation, elle est nécessairement composée. Il nous semble que Locke hésite sur ce point. En II, i, 25, il affirme que les idées simples sont les 《idées particulières des objets des sens 》; de même en III, iv, 16 , il montre que les «idées simples ont très peu de degré dans ce sens que les logiciens nomment Linea Praedicamentis $\gg ;{ }^{2 \cdot 2}$ mais en II, xxxi, 12, il soutient que l'esprit a trois sortes d'idées abstraites ou essences nominales, et il fait des idées simples la rremière espèce d'idées abstraites, les idées simples des qualités sensibles ne seraient en ce cas que des parties des données particulières de la sensation ${ }^{23}$. Il y a là un problème fondamental qui met

21. «Il ne parait pas que les idées confusos, comme celles que nous avons de la douceur, méritent ce nom. Car, quoiqu'elles expriment la puissance qui produit la sensation, elles ne l'expriment pas entièrement, ou du moins nous ne pouvons pas le savoir: car si nous comprenions ce qu'il y a dans cette idée que nous avons de la douceur, nous pourrions juger si elle est suffisante pout rendre raison de tout ce que l'expérience y fait remarquer ».

22. Locke semble supposer alors que les particuliers sont des idées simples: « La dernière espèce n'est qu'une seule idée simple, on n'en peut rien retrancher pour faire que ce qui la distingue des autres étant ôté, elle puisse convenir avec quelqu'autre chose $»$.

23. III, iii, 9, «Chaque terme plus général signifie une certaine idée qui n'est qu'une partie de quelqu'une de celles qui sont contenues sous elles». 
en cause la cohérence de la doctrine lockienne, ainsi que son rapport au rationalisme de type cartésien.

4 - Le chapitre $\mathrm{V}$ de l'ouvrage de monsieur Duchesneau, intitulé «Locke philosophe de l'expérience», est le plus important, parce que l'auteur y achève de déployer son interprétation de l'empirisme lockien. L'empirisme de l'Experimental Philosophy (Boyle) repose, selon monsieur Duchesneau, sur une circularité fondamentale : pour expliquer la naissance de la perception sensible de la qualité des choses on suppose une théorie atomistique de la matière, l'expérience doit fonder la science, mais un savoir a priori doit interpréter l'expérience ${ }^{24}$. Le but de l'auteur est de montrer comment Locke résoud le problème; une remarquable analyse (pp. 151 sq.) des trois textes où ce dernier traite de la notion de pouvoir (Essay, II, vii, 8; II, xxi ; II, xxvi, 1-2) introduit à cette solution. La notion de pouvoir est à la fois une idée simple de la réflexion (nous pouvons déterminer certaines parties de notre corps à se mouvoir), ou de la sensation (effet que les corps naturels sont capables de produire les uns sur les autres), et une idée complexe de mode simple (l'esprit considère en une chose la possibilité que n'importe quelle de ses idées simples soit changée, et dans une autre la possibilité d'opérer ce changement; le pouvoir est une relation). Il y a un paradoxe à considérer cette notion comme simple et comme composée; en fait, le pouvoir en tant que donnée sensible n'est pas réductible à une forme particulière du perceptible, il s'impose à travers toute perception, issue de la sensation comme de la réflexion; de plus, il est une donnée simple, car s'il implique toujours une relation à l'action productrice, il est manifesté

24. Cette remarque ne peut être un reproche que dans la perspective du rationalisme classique, c'est-à-dire dans l'hypothèse d'une philosophie réflexive gui se donne pour but de fonder la science. On soutiendrait volontiers aujourd'hui que ce rôle fondateur n'est qu'une illusion (cf. les célèbres analyses de Cavaillès in Logique et Théorie de la Science, P.U.F., 1960); on a, par ailleurs, proposé des classifications circulaires des sciences (Piaget) . Il importerait, à notre sens, de savoir dans quelle mesure ce thème du fondement est présent chez les empiristes classiques, et plus particulièrement chez Locke. La question historique fondamentale est en effet de déterminer si la philosophie lockienne de la connaissance est une réponse au problème du fondement, et si par conséquent monsieur Duchesneau a raison de l'interpréter dans ce sens. 
directement par tout effet sensible, tout phénomène perceptible, toute donnée de la perception. Dans ces conditions, ce qu'il faut c'est de chercher quelles sont les notions simples qui sont des notions d'effets, et comment elles révèlent les pouvoirs qui les produisent. L'idée est à la fois une perception de l'esprit et une modification de la manière dans le corps qui causent de telles perceptions en nous.

Mais il faut distinguer dans les corps les qualités premières et les qualités secondes: Locke affirme que l'on peut connaître les relations de ces qualités premières aux idées qu'elles produisent en nous; les qualités secondes apparaissent comme un simple pouvoir de déterminer une apparence sensible dans la conscience (couleurs, sons, goûts). Or Locke ajoute que c'est par les qualités premières que les corps produisent en nous ces phénomènes de conscience. Par là est attestée leur valeur objective, et le rapport des deux types d'idées, sans que soit remis en cause le phénoménisme emprunté à l'empirisme médical. L'hypothèse corpusculaire, en effet, n'est qu'une explication hautement probable des phénomènes naturels, mais il semble impossible d'en déduire rigoureusement les qualités sensibles des réalités naturelles, telles que nous pouvons les observer. C'est l'examen de nos idées des qualités sensibles qui nous permet de distinguer les qualités inhérentes aux réalités et celles qui surgissent secondairement de notre pouvoir de perception sensible, et du pouvoir d'interrelation des réalités naturelles. L'idée de pouvoir apparaît comme une caractéristique téléologique, expliquant l'interrelation des réalités naturelles et la possibilité pour le sujet conscient de la percevoir. Toutefois, monsieur Duchesneau note que la question de l'objectivité du savoir rationnel n'est pas totalement résolue ; tout savoir résulte en effet, pour Locke, d'une comparaison des idées lcs unes avec les autres: le rationalisme cartésien ne disait pas autre chose, mais il fondait la rationalité dans la conception claire et distincte des natures simples. Après avoir rappelé la fameuse définition de la sémiotique ou science des signes (mots et idées) du livre IV, c'est dans la doctrine lockienne du langage que notre auteur tente de trouver une réponse au problème du fondement de la rationalité (i.e. de l'objectivité du savoir rationnel conçu comme des rapports inter-idées). En s'appuyant sur les travaux 
de M. Aarsleff, ${ }^{25}$ Amstrong, ${ }^{26}$ Yolton, ${ }^{27}$ monsieur Duchesneau montre que les analyses linguistiques de Locke se situent strictement sur un plan épistémologique, et qu'elles écartent la recherche métaphysique concernant le rapport de signification; sa doctrine conventionaliste de l'origine des mots est un simple postulat épistémologique, qui se borne à la synchronie, et refuse l'analyse diachronique où Leibniz en situera la critique. Si le rapport de signification qui lie le mot à l'idée est toujours arbitraire, cette référence directe et immédiate ne se suffit pas à elle-même et implique le rapport de l'idée à une cause productive $;{ }^{28}$ au centre de toute l'analyse se trouve l'adéquation du rapport de signification avec l'intelligibilité même du réel. Selon monsieur Duchesneau, «la sémiotique apparaît comme la seule doctrine de la rationalité conciliable avec l'analyse empiriste des idées» (p. 203) ; cette conclusion repose sur la thèse que le signe (qu'il soit mot ou idée) inclut toujours en lui-même sa référence objective, et que par là la sémiotique intériorise dans la fonction signifiante elle-même le rapport de finalité qui lie la connaissance au monde. Cette thèse, l'auteur la fonde en dernier lieu sur l'interprétation d'un texte fondamental concernant la vérité ; celle-ci nous semblant contestable, c'est par sa discussion que nous introduirons la mise en perspective critique de tout l'ouvrage.

5 - Le terme vérité ne semble signifier pour Locke «rien d'autre que le fait d'unir ou de séparer des signes de la même façon que les choses qu'ils signifient, conviennent ou non l'une avec l'autre. Le fait d'unir ou de séparer des signes, dont il s'agit ici, est ce que nous appelons autrement proposition. Ainsi, la vérité appartient seulement aux proposition, dont il

25. AARSLEFF, $\mathrm{H}$. «Leibniz on Locke on Language», in American Philosopbical Quaterly, I (1964), pp. 165-186.

26. ARMSTRONG, R.L. "John Locke's Doctrine of Signs: A new Metaphysics 》 Journal of the History of Ideas, XXVI (1965), pp. 369-382.

27. YOLTON, Locke and the Compass of Human Understanding, Cambridge, C.U.P. 1970 .

28. Il nous semble qu'une erreur typographique s'est glissée p. 201; on lit «le rapport de signification, qui implique que la relation nom-idée soit toujours dépendance par rapport à une relation nom-chose; » nous croyons que monsieur Duchesneau a voulu écrire; "Soit toujours dépendante par rapport à une relation idée-chose». 
existe deux sortes: à savoir les propositions verbales et les propositions mentales, de même qu'il y a deux sortes de signes communément en usage: les idées et les mots ${ }^{29}$. Les implications que monsieur Duchesneau tire de cette définition indiquent assez l'interprétation qu'il lui donne:

(i) le critère de la vérité cesse d'être formel et devient totalement matériel, c'est l'accord ou la convenance des choses entre elles; la vérité des propositions ne réside que dans les rapports objectifs qui les sous-tendent, et que l'expérience seule peut manifester.

(ii) la théorie représentationaliste des idées est réfutée: si le critère de la vérité est l'accord des réalités elles-mêmes, c'est que l'idée n'est pas l'objet exclusif de l'acte de perception, l'idée n'est pas seulement une copie mentale d'un objet, elle est à la fois cette image et sa référence objective, ou raison d'être ou raison signifiante.

(iii) se trouve expliquée une aporie apparente du livre IV; Locke y soutient en effet que connaître c'est percevoir un rapport entre idées, et il range l'existence réelle parmi ces relations, or un rapport d'existence réelle ne semble pas pouvoir se réduire à une relation entre deux idées (celle d'un sujet et celle d'une existence) ${ }^{30}$.

Il ne nous semble pas que le texte le Locke réduise la vérité à un rapport entre des réalités, il nous paraît, au contraire, qu'il reprend la doctrine très classique selon laquelle une proposition est vraie lorsqu'elle relie des termes qui sont réellement unis ou lorsqu'elle sépare des termes réellement séparés; autrement dit, le vrai réside, non dans l'accord des choses entre elles, mais dans la correspondance de l'image à la réalité ${ }^{31}$. Si notre interprétation est correcte, la logique lockienne utilise dans sa théorie de la vérité

29. IV, $v, 2$.

30. Monsieur Duchesneau est ici en accord avec Bréhier, qui fait la même remarque 1. c. .p 254.

31. Cf. ARISTOTE, Métapbysique, E. 4, 1027 b 18, et A, 10, 1051 b 2: "être dans le vrai c'est penset que ce qui est séparé est séparé, et ce qui est uni est uni, et être dans le faux, c'est penser contrairement à la nature des objets $\gg$. 
deux niveaux sémantiques distincts: celui des idées (convenance entre deux idées), celui des choses (convenance de l'idée à la chose). C'est bien ce qui ressort de la distinction faite entre la vérité verbale et la vérité réelle; au premier niveau sémantique, «cette proposition, tous les centaures sont des animaux sera aussi vraie que celle-ci tous les bommes sont des animaux;》 mais «quoique les mots ne signifient autre chose que nos idées, (...) ils sont destinés à signifier des choses, ${ }^{32}$ c'est pourquoi doit intervenir le second niveau sémantique pour définir la vérité réelle. «Les propositions renferment une vérité réelle lorsque les signes dont elles sont composées sont joints suivant que nos idées conviennent, et lorsque ces idées sont telles que nous ne connaissons capables d'exister dans la nature, ce que nous ne pouvons connaitre à l'égard des substances, qu'en sachant qu'il en a existé de telles ${ }^{33}$. La question qui se pose alors est celle des critères de correspondance des idées au réel; Locke tente de la résoudre au niveau de la doctrine des idées complètes et incomplètes (adequate and inadequate). L'idée simple est nécessairement complète, c'est-à-dire qu'elle a valeur objective ; nous teconnaissons volontiers que Locke fonde cette objectivité sur la finalité (rapport que Dieu a mis entre nos idées des qualités sensibles et ces qualités) ; le problème est plus délicat en ce qui concerne les idées complexes; celles-ci en effet sont arbitraires, «étant des combinaisons que l'esprit joint ensemble par un libre choix; ${ }^{34}$ la question est résolue de façon purement verbale : «toutes les idées de cet ordre sont elles-mêmes regardées comme des archétypes, et les choses ne sont considérées qu'en tant qu'elles $y$ sont conformes ${ }^{35}$. Il faudrait en effet expliquer comment le rapport archétypal des idées aux choses est possible, et s'il ne ramène pas à l'idéalisme cartésien; lorsque Locke essaie de montrer que "notre connaissance n'est pas moins véritable ou certaine, parce que les idées de morale sont de notre propre invention, et que c'est nous qui leur donnons des noms», il se fonde sur une comparaison avec l'arbitraire du langage et sur ce qu'appeler triangle un trapèze ne change rien à une démonstration; mais puis-

32. IV, $\mathrm{v}, 7$.

33. Ibid. 8.

34. IV, iv, 4. 
que dans le cas considéré, la vérité provient de la seule convenance des idées, et que ces idées sont des groupements arbitraires d'idées simples, on ne voit pas comment cette convenance pourrait être autrement qu'arbitraire ${ }^{36}$. C'est toute la morale et toute la mathématique qui tombent. Cette interprétation implique que, contrairement à la thèse de monsieur Duchesneau, il y ait chez Locke une théorie représentionaliste de l'idée; l'idée possède une double réalité : elle est un élément de l'esprit et une représentation, c'est-à-dire une image; c'est parce que les idées sont des images qu'on peut les manier comme des signes de la réalité, en supposant incluses dans leurs propriétés intrinsèques leurs possibilités de référence objective. Il s'ensuit que le paradoxe du traitement de l'existence dans le livre IV demeure; nous pensons qu'il est inhérent à la philosophie lockienne. Monsieur Duchesneau avance que l'existence réelle ne peut être une relation que si on suppose que l'intelligibilité du réel est contenue dans le rapport de l'idée signe au monde, il exclut, comme Bréhier, que l'existence soit une idée (un prédicat) susceptible de convenir ou non avec un sujet. Ceci va à l'encontre de l'interprétation que Leibniz donne de ce passage; personne au XVIIe siècle ne soutient que l'existence n'est pas un prédicat. Locke lui-même ne peut le faire car, puisque nos propositions linguistiques n'expriment que la convenance ou disconvenance de deux idées, la proposition «Dieu existe » exprime la convenance de l'idée de Dieu à celle de l'existence. Cette position, en outre, est cohérente avec le thème d'une démonstration de l'existence de Dieu. Ou celui d'une connaissance intuitive de notre existence, puisque l'intuition est définie comme la perception immédiate de la convenance de deux idées. Elle est par contre en désaccord avec l'affirmation que les propositions générales ne concernent pas

35. Ibid., 9; seul, à l'époque, Leibniz a abordé ce genre de problème de de façon relativement satisfaisante, parce qu'il distinguait la réalité des rapports et l'arbitraire des noms par lesquels on désigne les termes de ces rapports; cf. Dialogues, de connexione inter res et verba (1677), édition Gerhardt, tome VII, p. 190 sq.

36. On trouve chez Locke la thèse selon laguelle les idées complexes nous sont imposées par notre contact avec le monde externe (par exemple la conjonction constante dans le cas des idées complexes de substances) ; mais cela est peu compatible avec l'affirmation de l'arbitraire. 
l'existence, ${ }^{37}$ puisque l'idée d'existence n'est qu'un terme géné. ral, et avec le fait que seule la sensation nous apporte la connaissance de l'existence des choses extérieures, ${ }^{38}$, puisqu'alors le rapport d'existence réelle est véritablement inclus dans l'acte de sensation.

6 - Nous serons d'accord avec monsieur Duchesneau pour accorder dans la théorie lockienne de la connaissance une place centrale à la sémiotique. On peut considérer que cette troisième division du savoir, à côté de la pratique et de la physique, recouvre, en fait, tout le contenu de l'Essay; en ce sens, c'est une théorie de la pensée, du langage et du raisonnement. On attendrait alors, plutôt que le dernier chapitre sur la critique lockienne de la vision en Dieu, une étude critique de cette sémiotique. Il nous semble, en effet, qu'elle est lourde d'ambiguité; si, par exemple, on y trouve une théorie de l'idée signe et du mot signe, on ne rencontre jamais de définition du mot signe qui soit explicite; or un mot et une idée peuvent-ils être signes au même titre? L'idée semble plutôt un signe iconique et le mot un signe arbitraire ${ }^{39}$. En fait, au sein même des difficultés que nous avons soulignées ici, ce qui apparaît, c'est l'ambiguïté de l'empirisme lockien. La philosophie lockienne brasse assurément des thèmes d'origine rationaliste et idéaliste; c'est le cas, par exemple, de la théorie de l'inférence par intuition de la convenance entre deux idées, dont monsieur Duchesneau note qu'elle est en tout point cartésienne. Mais, à l'inverse, on y rencontre le thème de l'irréductibilité du jugement d'existence à la démonstration (excepté pour Dieu), et c'est cet aspect du lockisme que les Lumières retiendront; la polémique avec Worcester révèle que, quand bien même nous n'avons pas d'idée claire d'une chose, il

37. IV, ix, 1.

38. Ibid. IV. xi.

39. Le chap. VI du livre de D.J. O'Connor Jobn Locke (Dover, 1966) traite de façon intéressante de ces problèmes dans la perspective de la philoso. phie analytique; bien que cet ouvrage passe sous silence bien des difficultés (p. 140, O'Connor parle d'idées générales complexes, or en II, xi, 9, Locke affirme que les idées générales sont simples), on s'étonne que monsieur Duchesneau ne le cite pas dans sa bibliographie. 
ne s'ensuit pas qu'elle n'existe pas; parmi les arguments, on trouve référence faite à la gravitation newtonnienne. Si on sait que Locke, comme le fait remarquer Leibniz, n'a jamais changé le passage de l'Essay (II, vii, 11) où il affirme que les corps opèrent par impulsion, et non autrement, on ne peut que noter la convergence de sa théorie du raisonnement appliqué à la méthodologie scientifique, avec la nouvelle philosophie naturelle, ${ }^{40}$ et par conséquent son opposition radicale au cartésianisme. En critiquant l'argument de Malebranche, selon lequel le processus naturel de la connaissance consiste à passer de la considération de tous les êtres d'un point de vue général à la vision particulière de celui que nous désirons apercevoir, Locke pose la dépendance de l'abstrait et du général par rapport aux données particulières de l'expérience sensible pour la conscience, monsieur Duchesneau le note excellemment. Mais alors, ne doit-on pas dire que, loin que la sémiotique apparaisse la seule doctrine de la rationalité conciliable avec l'analyse empiriste des idées, c'est au contraire la genèse empiriste des idées qui assure à la sémiotique, conçue comme logique, sa rationalité, c'est-à-dire son rapport au réel ? A dire le vrai, ni cette thèse ni la thèse inverse, proposée par monsieur Duchesneau, ne nous semblent totalement satisfaisantes. La doctrine lockienne est polyvalente; plus précisément la théorie de la connaissance nous paraît relever de deux courants distincts, selon qu'elle s'applique à Dieu, à la morale et aux mathématiques, ou qu'elle concerne le monde externe. La signification de l'empirisme lockien est ambiguë, et cette ambiguité ne peut être comprise qu'au travers d'une étude précise de la sémiotique. Celleci est sans conteste la métaphysique nouvelle que d'Alembert attribue à Locke dans le discours préliminaire de I'Encyclopédie, et on la comprendra sans doute mieux en la rapportant à l'image qu'en ont forgée les Lumières ${ }^{41}$.

Que cette image ne soit pas la seule possible indique alors le rôle historique charnière que son ambiguïté même confère

40. Voir note 2.

41. Certains thèmes de l'Essay sont sélectionnés; ce n'est sans doute pas un hasard si un des deux articles «propositions》 de l'Encyclopédie est une reprise textuelle de passage des chap. VII et VIII du livre IV. 
au lockisme, ${ }^{42}$ et l'intérêt essentiel qu'on peut trouver dans la lecture d'un ouvrage aussi bien informé que celui de monsieur Duchesneau.

\section{APPENDICE}

Ce compte rendu était déjà rédigé lorsque monsieur Duchesneau nous a fait parvenir un article sur « Locke et le savoir de probabilité ${ }^{43}$ qu'il a écrit quelque temps après son ouvrage. En notant qu' «il y a lieu de supposer que, si les idées sont considérées comme des signes des réalités naturelles, ces signes ont une raison d'être dans la nature des choses, et qu'une association de ces signes conforme à la nature traduirait de façon suffisamment intelligible, la logique inhérente aux phénomènes, tels qu'ils sont perçus » (p. 190), il nous semble que l'auteur nuance ses positions concernant la représentationalité ${ }^{4}$ des idées. $\mathrm{Pa}$ reillement lorsqu'il remarque que «l'application de la méthode descriptive implique que le problème des fondements de la probabilité ne soit pas intrinsèquement distingué de celui des degrés de la probabilité »(p. 191), il nous semble qu'il saisit la problématique empiriste hors des présupposés rationalistes qu'on pouvait lui reprocher dans son ouvrage. La question du rapport à Newton, et aux Lumières (en l'occurence d'Alembert) est aussi absordée, d'une façon qui nous fait souhaiter que monsieur Duchesneau, qui apparaît actuellement comme l'un des meilleurs spécialistes de Locke, approfondisse la question dans des études ultérieures.

Université de Rouen (France)

42. Dans la perspective de monsieur Duchesneau, une étude comparé avec la notion condillacienne d'analyse permettrait sans doute de compléter la compréhension du rôle que joue la finalité dans les doctrines empiristes de la connaissance.

43. Dialogue, vol. XI, 1972, pp. 185-203.

44. En fait le problème nous paraît reposer sur une ambiguïté - que l'idée représente la chose pourrait vouloir dire qu'elle en est une image ressemblante. C'est ce que Locke nie expressément (cf. II, viii, 7). Une relation de ressemblance entre une idée a et une chose b, peut s'expliciter dans le fait que l'idée et la chose ont au moins une propriété en 
commun, c'est-à-dire / $a R b \Leftrightarrow[\mathrm{ff}(\mathrm{a}) . \mathrm{f}(\mathrm{b})] . /$ Si on nie ce point il ne s'ensuit pas qu'on refuse d'expliciter la relation de signe. Si, sous la forme de propriétés conjointes; pratiguement on pose souvent quelque chose comme $/ \mathrm{a} S^{\mathrm{b}} \mathrm{b} \Leftrightarrow[\mathrm{f}(\mathrm{a}) \cdot \mathrm{g}(\mathrm{b})]$, / ainsi dans tel cas $\mathrm{a} \mathrm{S}_{\mathrm{b}} \mathrm{b}$ est équivalent au fait que a soit un nom propre et $b$ un individu. Cela est vrai pour toute théorie qui ne réduit pas l'analyse logique à un travail portant sur les seules propriétés des expressions (on pourrait dire que Locke opère une telle réduction et qu'il analyse des différences entre les idées de gualités sensibles, de mode, de substance ou de relation au seul niveau des propriétés de ces idées) mais on ne s'en tient pas à une telle réduction, si on se pose certaines restrictions destinées à nous garantir que les idées ne sont pas de signes vides; pour se faire on assume l'existence des idéats; ainsi ${ }_{a} S_{\mathfrak{b}} b$ peut impliquer que a est une idée complète et $b$ une qualité sensible, ou a une idée incomplète et $b$ une substance. Locke ne pose ces proprićtés conjointes que pour justifier une doctrine de la vérité qui soutient comme méthathéorème " la proposition 《 $\mathrm{p}$ » est vrai si $\mathrm{p}$ ». La proposition $\mathrm{p}$ exprime le rapport $C$ de convenance entre les idées $a$ et $b$, elle est vraie s'il y a le rapport réel $C_{1}$, entre les idéts $a_{1}$ et $b_{1}$; dès lors $a C b$ est vrai, si $\quad a_{1} C_{1} b_{1} ; \quad$ or la théorie des idées complètes et incomplètes permettrait dans certains cas de conclure si on $\mathrm{aS}_{\mathrm{a}_{1}}, \mathrm{bS}_{1} \mathrm{~b}_{1}, \mathrm{aCb}$, que $\mathrm{a}_{1} \mathrm{C}_{1} \mathrm{~b}_{1}$ est un fait. En tout état de cause si l'on peut admettre que les idées ne sont pas représentatrices des choses au sens d'images ressemblantes, il ne semble pas qu'on puisse en dire autant des propositions qui conservent au moins la structure formelle des faits. La doctrine lockienne des idées négligeant la forme, ne permet pas une discussion correcte de ce problème; dans ces conditions, si on voulait nier que nos pensées soient en quelque façon des images ressemblante; des choses, il faudrait faire comme Hume dans le cas des impressions, c'est-à-dire refurer d'aller au-delà, ce qui ne paraît pas cohérent avec le réalisme lockien. 